\title{
Steady and Unsteady Nozzle Simulations Using the Conservation Element and Solution Element Method
}

\author{
David J. Friedlander ${ }^{1}$ and Xiao-Yen Wang ${ }^{2}$ \\ NASA Glenn Research Center, Cleveland, $\mathrm{OH}, 44135$
}

\begin{abstract}
Time-accurate, Euler, quasi-1D and 2D-axisymmetric computational fluid dynamic (CFD) simulations of a three-stream plug nozzle were performed as part of an effort to provide an efficient and accurate CFD-based approach to modeling nozzle dynamics. The CFD code used for the simulations is based on the space-time Conservation Element and Solution Element (CESE) method. Steady-state results were validated using the Wind-US code and a code utilizing the MacCormack method while the unsteady results were partially validated via an aeroacoustic benchmark problem. The CESE steady-state flow field solutions showed excellent agreement with solutions derived from the other methods and codes while preliminary unsteady results for the three-stream plug nozzle are also shown. Additionally, a study was performed to explore the sensitivity of gross thrust computations to the control surface definition. The results showed that most of the sensitivity in computing the gross thrust is attributed to the control surface stencil resolution and choice of stencil end points and not to the control surface definition itself. Finally, comparisons between the quasi-1D and 2D-axisymetric solutions were performed in order to gain insight on whether a quasi-1D solution can capture the steady and unsteady nozzle phenomena without the cost of a $2 \mathrm{D}$-axisymmetric simulation. Initial results show that while the quasi1D solutions are similar to the 2D-axisymmetric solutions, the inability of a quasi-1D simulation to predict two dimensional phenomena limits its accuracy.
\end{abstract}

\section{Nomenclature}

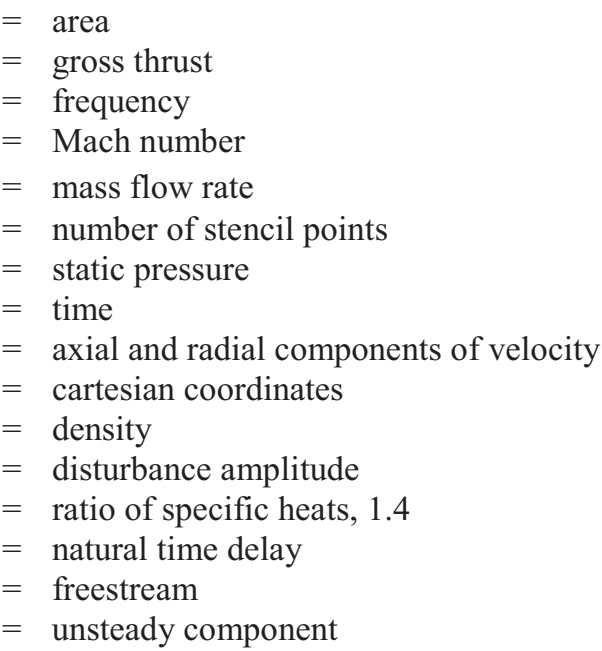

\section{Introduction}

s research and development budgets become more constrained with time, wind-tunnel model testing becomes less affordable for aircraft design. Computer-based models have the potential to enable rapid aircraft design

\footnotetext{
${ }^{1}$ Aerospace Engineer, Inlet and Nozzle Branch, 21000 Brookpark Road, MS 5-12, AIAA Member.

${ }^{2}$ Aerospace Engineer, Thermal Systems Branch, 21000 Brookpark Road, MS 86-12, AIAA Member.
} 
and analysis at reduced expense compared to wind-tunnel model testing. This is exceptionally true in the early and intermediate stages of the design phase. As such, there are efforts in the aerospace industry to develop models that maximize accuracy while minimizing the turnaround time and the computational resources needed. The model can encompass the entire aircraft, a particular system, or even a subcomponent of a system. This paper presents analyses of a simulated three-stream plug nozzle and investigates both steady and unsteady nozzle flow behaviors. The analyses encompassed quasi-1D and 2D-axisymmetric time-accurate Euler simulations. One of the primary goals of the simulations was to develop an optimized computational fluid dynamic (CFD)-based nozzle model that could be used as part of a system level model. In this paper, optimization is defined as minimizing the run time and the amount of computational resources needed to compute the time-varying gross thrust from a nozzle while retaining an accurate prediction. This paper compares the results of the simulations and also explores factors involved in calculating gross thrust for a CFD-based nozzle model.

\section{Geometry and Numerical Modeling}

The nozzle chosen for the CFD simulations consists of three internal ducts with a downstream conical plug or center body. For nomenclature clarity the ducts are denoted as follows (in increasing radial order): inner duct, primary duct, and noise shielding (or simply shield) duct. The simulations used quasi-1D and 2D-axisymmetric grids to represent the nozzle. Due to the proprietary nature of the actual nozzle, this report will only show a generic layout of the nozzle, shown in Fig. 1, and present results in a non-dimensional format based on the freestream density, speed of sound, and a characteristic length.

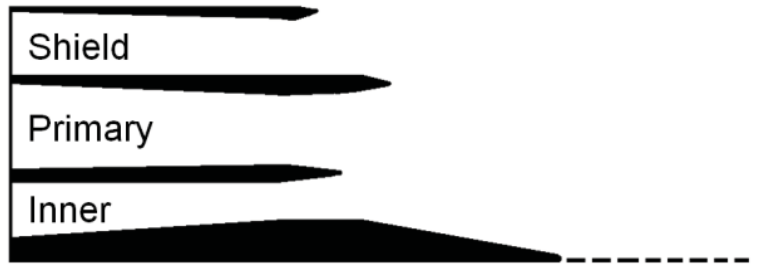

Figure 1. Generic three-stream nozzle geometry (axisymmetric cut).

The space-time Conservation Element and Solution Element $(\mathrm{CESE})^{1,2,3}$ method, originally proposed by Chang at NASA's Glenn Research Center, was the primary numerical method chosen for the CFD simulations. The CESE method is a time accurate formulation with flux-conservation in space-time and utilizes a staggered mesh. The staggered mesh allows for the scalar quantities (e.g. pressure, density) to be computed at the cell center while the vector flow quantities (e.g. momentum, velocity) are computed at the cell faces. One benefit of this is that it eliminates the need to solve the Riemann type problem along the cell interfaces. Further, the CESE method is $2^{\text {nd }}-$ order accurate in both space and time with the stability condition of Courant-Friedrichs-Lewy (CFL) number less than one. In the past, the CESE method has been applied to a wide variety of CFD and computational aeroacoustic (CAA) problems ${ }^{4,5,6}$. The CESE method has been proven to be a robust and efficient tool for dynamic simulations and aero-acoustic computations.

In addition to the CESE method, a code utilizing the MacCormack scheme ${ }^{7,8}$ and the Wind-US code ${ }^{9}$ were chosen to help validate the CESE derived steady-state flow fields. The MacCormack scheme was chosen for its simplicity in modeling a quasi-1D flow field. However, the chosen MacCormack scheme-based code only allows for quasi-1D simulations. The Wind-US code was then chosen for comparing the 2D-axisymmetric simulations and has been shown to model nozzle flows effectively ${ }^{10}$. Wind-US is a general purpose compressible flow solver developed by the NPARC Alliance that can run in either Reynolds-Averaged Navier-Stokes (RANS) or Euler modes. The default scheme for Wind-US is Roe's $2^{\text {nd }}$-order up-winding scheme ${ }^{11,12}$ and unless otherwise specified, was run in the Euler mode.

\section{Flow Field Correlation and Validation}

\section{A. Steady-State Quasi-1D Simulations}

The CESE quasi-1D Euler code was used to compute a steady-state solution for each duct of the nozzle. Each duct was represented by its own 200 uniform-spaced element grid. Two additional solutions were computed for comparison and validation: a quasi-1D, perfect gas, isentropic analytical solution and an independent quasi-1D 
solution utilizing the MacCormack scheme, both provided by Mr. Joseph Connolly. Results from these simulations for the primary duct are shown in Fig. 2. It can be seen that the CESE solution is in excellent agreement with the MacCormack and analytical solutions for the steady-state flow field.
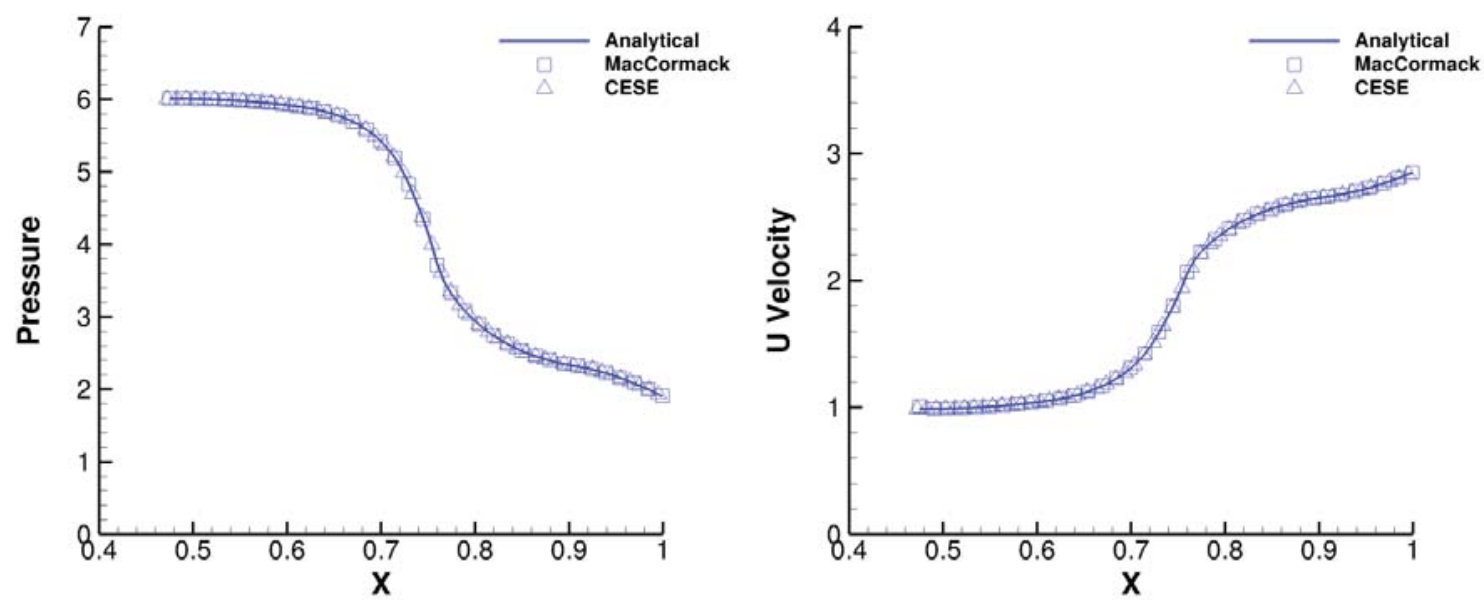

Figure 2. Quasi-1D pressure (left) and $u$ velocity (right) axial distributions for the primary duct.

\section{B. Steady-State 2D-Axisymmetric Simulations}

2D-axisymetric simulations were performed to assess the limitations of the lower resolution quasi-1D simulations. Two codes were used: the 2D CESE Euler code and Wind-US. The CESE simulations used a constant time step while the Wind-US simulations used a constant CFL number. For the Wind-US simulation, a 129,000 cell structured grid modeled the external plume and plug regions as well as all three internal ducts of the nozzle. The CESE simulation used a diagonalized derivative of the Wind-US grid, resulting in a 258,000 cell triangular grid. An example of the diagonalization process is shown in Fig. 3.

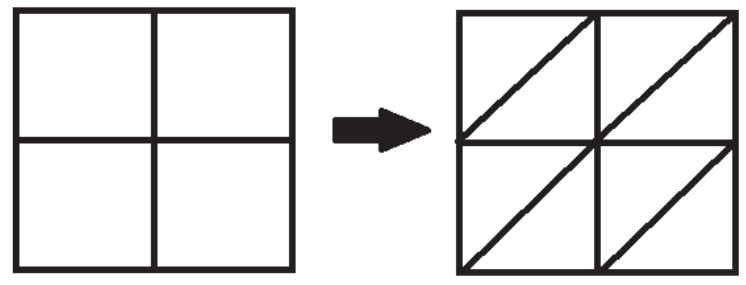

Figure 3. Example of diagonalizing a structured grid.

A grid resolution study was performed on three levels of refinement of the Wind-US grid. The coarse level used every fourth grid point in each computational direction, the medium level used every other grid point in each computational direction, and the fine level used all grid points. The mass flow rate through the mixing region of the inner and primary ducts was computed via the Wind-US utility code CFPOST at each grid level, the results of which are shown in Table 1. Note that the percent difference between the coarse and medium grid levels (c-m) was defined as:

$$
\% \text { Difference }=100 \times \frac{\dot{m}_{\text {coarse }}-\dot{m}_{\text {medium }}}{\dot{m}_{\text {medium }}}
$$

The percent difference between the medium and fine grid levels $(m-f)$ was defined as:

$$
\% \text { Difference }=100 \times \frac{\dot{m}_{\text {medium }}-\dot{m}_{\text {fine }}}{\dot{m}_{\text {fine }}}
$$


while the percent mass flow gained was defined as:

$$
\% \text { MassFlow }=100 \times \frac{\dot{m}_{\text {Zone }, \text { i=last }}-\dot{m}_{\text {Zones }+ \text { Zone6 } 6}}{\dot{m}_{\text {Zones }+ \text { Zone6 }}}
$$

Figure 4 shows a graphical representation of the grid zones presented in Table 1. It can be seen from Table 1 that the fine grid level is reaching grid convergence and is sufficiently converged for the presented analyses. Assured of grid convergence, $u$ velocity and pressure through the primary duct are shown in Fig. 5 for the 2D-axisymmetric Euler simulations. Note that the 1D cuts shown in Fig. 5 were taken as the streamline originating at the duct inlet, center height, to reduce the dimensionality for later comparisons with the quasi-1D solution. The streamline approach was used over an averaging technique to better capture the presence of shock waves. From Fig. 5 it is shown that the CESE solution agrees well with the Wind-US solution (within $0.407 \%$ for pressure and $0.163 \%$ for $u$ velocity). Both solutions show a sharp increase in pressure (with a corresponding decrease in $u$ velocity) near $x=0.9$, which is due to an oblique shock wave off one of the duct splitters. However, the CESE solution predicts the shock location just upstream of the Wind-US solution, resulting in a higher minimum pressure and a lower maximum $u$ velocity.

Table 1. Mass flow rates at various grid levels for the Euler Wind-US grid.

\begin{tabular}{|l|c|c|c|c|c|}
\hline \multicolumn{1}{|c|}{ Zone } & Coarse & Medium & Fine & \% Difference (C-M) & \% Difference (M-F) \\
\hline Zone 5 + Zone 4 (i=last) & 2.413 & 2.401 & 2.398 & 0.500 & 0.125 \\
\hline Zone 6 (i=1) & 2.379 & 2.393 & 2.401 & -0.585 & -0.333 \\
\hline Zone 6 (i=last) & 2.400 & 2.401 & 2.406 & -0.042 & -0.208 \\
\hline \% Mass Flow Gained & -0.539 & 0.000 & 0.334 & - & - \\
\hline
\end{tabular}

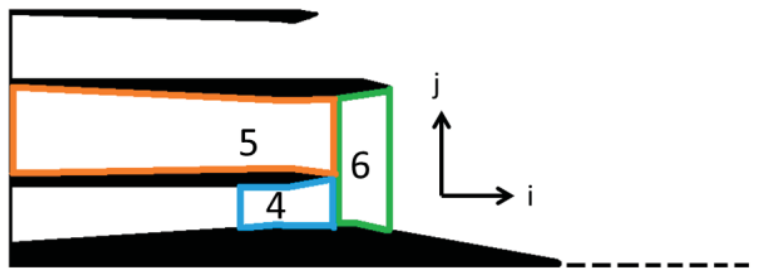

Figure 4. Graphical representation of the grid zones used in Table 1. Note that the number is the zone number.
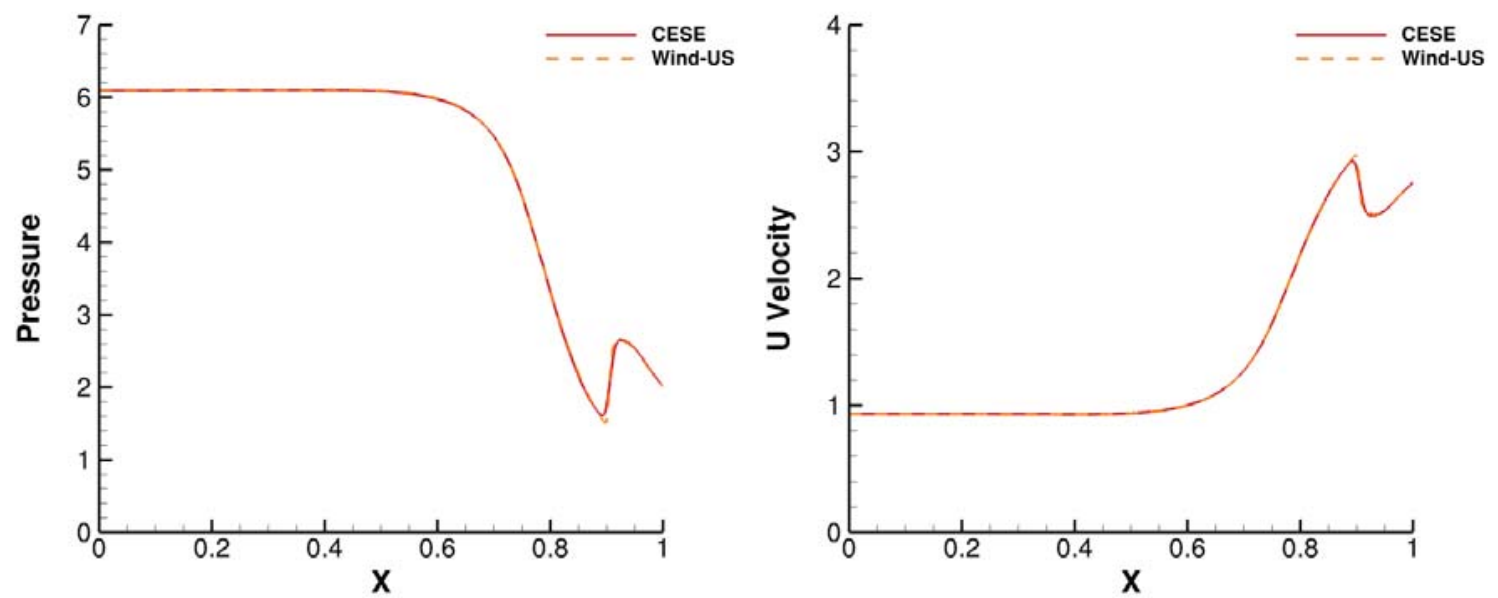

Figure 5. 2D-axisymmetric pressure (left) and $u$ velocity (right) axial distributions for the primary duct.

\section{Dynamic Quasi-1D Simulation}


For ease of validating an Euler, quasi-1D, unsteady flow field, the CESE method was applied to an aeroacoustic benchmark problem ${ }^{13}$ rather than directly to the nozzle of interest. The aeroacoustic benchmark problem simulated a downstream running acoustic wave at the inlet of a convergent-divergent nozzle with the acoustic wave disturbance defined as:

$$
\left[\begin{array}{l}
\rho^{\prime} \\
u^{\prime} \\
p^{\prime}
\end{array}\right]=\left[\begin{array}{l}
1 \\
1 \\
1
\end{array}\right] \varepsilon \sin \left(\omega\left(\frac{x}{1+M}-t\right)\right)
$$

where

$$
\begin{gathered}
{\left[\begin{array}{l}
\rho \\
u \\
p
\end{array}\right]=\left[\begin{array}{l}
\rho_{\text {steady }} \\
u_{\text {steady }} \\
p_{\text {steady }}
\end{array}\right]+\left[\begin{array}{l}
\rho^{\prime} \\
u^{\prime} \\
p^{\prime}
\end{array}\right]} \\
\varepsilon=1 \mathrm{E}-5 \\
\omega=0.6 \pi
\end{gathered}
$$

Note that the benchmark problem was poised in a non-dimensional format and the mean flow boundary conditions are shown in Table 2, per reference 13. Although the problem is over specified for a subsonic inflow, it is still a useful benchmark problem with steady and unsteady solutions shown in Fig. 6 and Fig. 7.

Table 2. Non-dimensional mean flow boundary conditions for the aeroacoustic benchmark problem

\begin{tabular}{|c|c|}
\hline $\boldsymbol{M}_{\text {inlet }}$ & 0.2006533 \\
\hline $\boldsymbol{p}_{\text {inlet }}$ & $1 / \gamma$ \\
\hline $\boldsymbol{\rho}_{\text {inlet }}$ & 1 \\
\hline $\boldsymbol{p}_{\text {exit }}$ & 0.6071752 \\
\hline
\end{tabular}

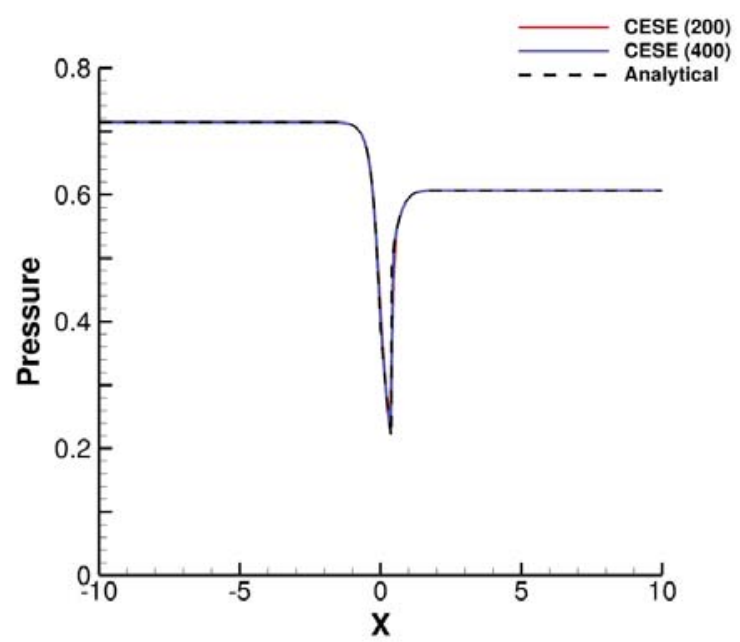

Figure 6. Steady-state pressure distribution. Note that the numbers in parenthesis are the number of grid elements. 

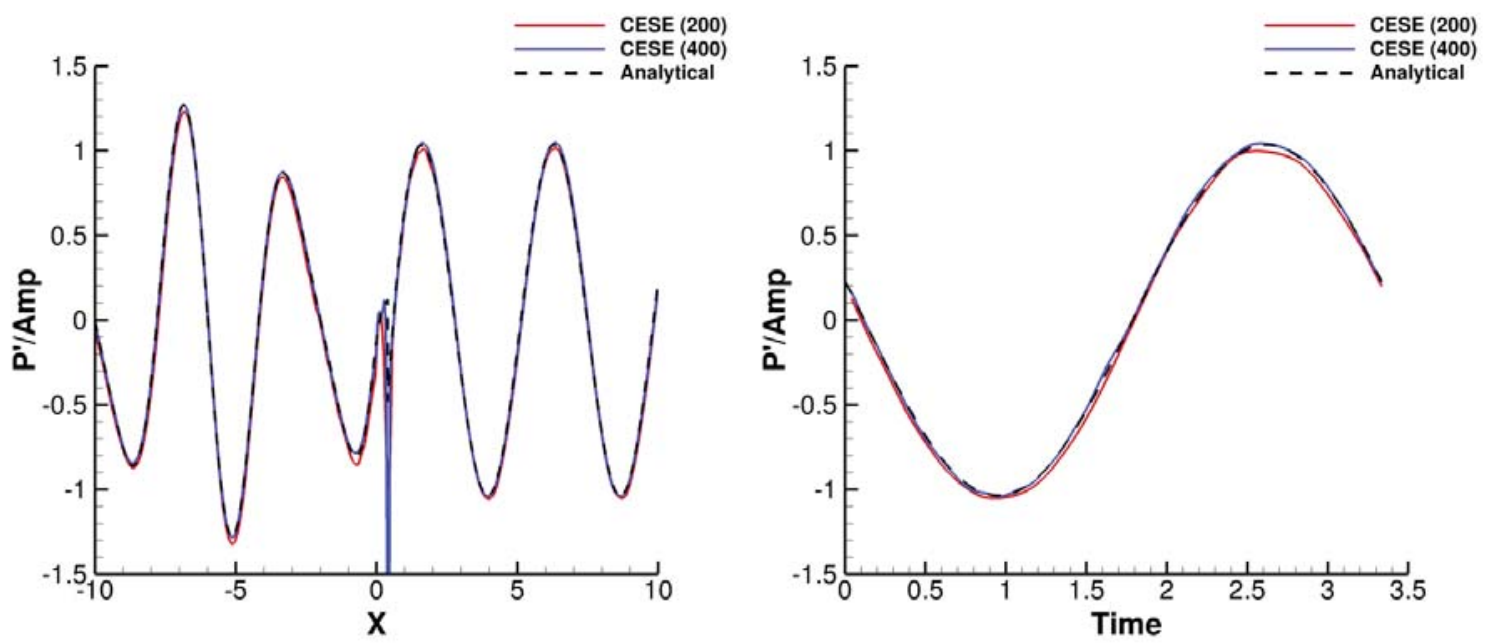

Figure 7. Dynamic pressure distribution (left) and time history at the nozzle exit for one cycle (right). Note that the numbers in parenthesis are the number of grid elements.

The results, computed using both 200 and 400 uniformly-spaced element meshes, show excellent agreement with the analytical solutions provided for the benchmark problem and serves as confirmation of the results shown by Wang et. al. ${ }^{14}$, who previously demonstrated success in modeling the quasi-1D steady-state flow field as well as the timevarying dynamics of the aeroacoustic benchmark problem with the CESE method. While this validates the CESE quasi-1D code, the authors are not aware of an equivalent benchmark problem to analytically validate a $2 \mathrm{D}-$ axisymmetric unsteady simulation.

\section{Comparisons Between Quasi-1D and 2D-Axisymmetric Simulations}

\section{A. Steady-State Flow Field}

After validating the CESE steady-state solutions, the 2D-axisymmetric solution was compared to the quasi-1D solution, shown in Fig. 8, to identify any differences in the computed flow fields. The quasi-1D solution agrees well with the $2 \mathrm{D}$-axisymmetric solution up to about $x=0.7$, with the quasi-1D solution slightly over predicting the upstream $u$ velocity (and subsequently under predicting the upstream pressure). However, the quasi-1D solution fails to pick up the oblique shock wave computed in the 2D-axisymmetric solution, which is a primary deficiency of a quasi-1D simulation. The oblique shock wave is represented by the sharp increase in pressure and decrease in $u$ velocity at $x=0.9$ and is the main reason for the discrepancy between the solutions downstream of $x=0.7$.
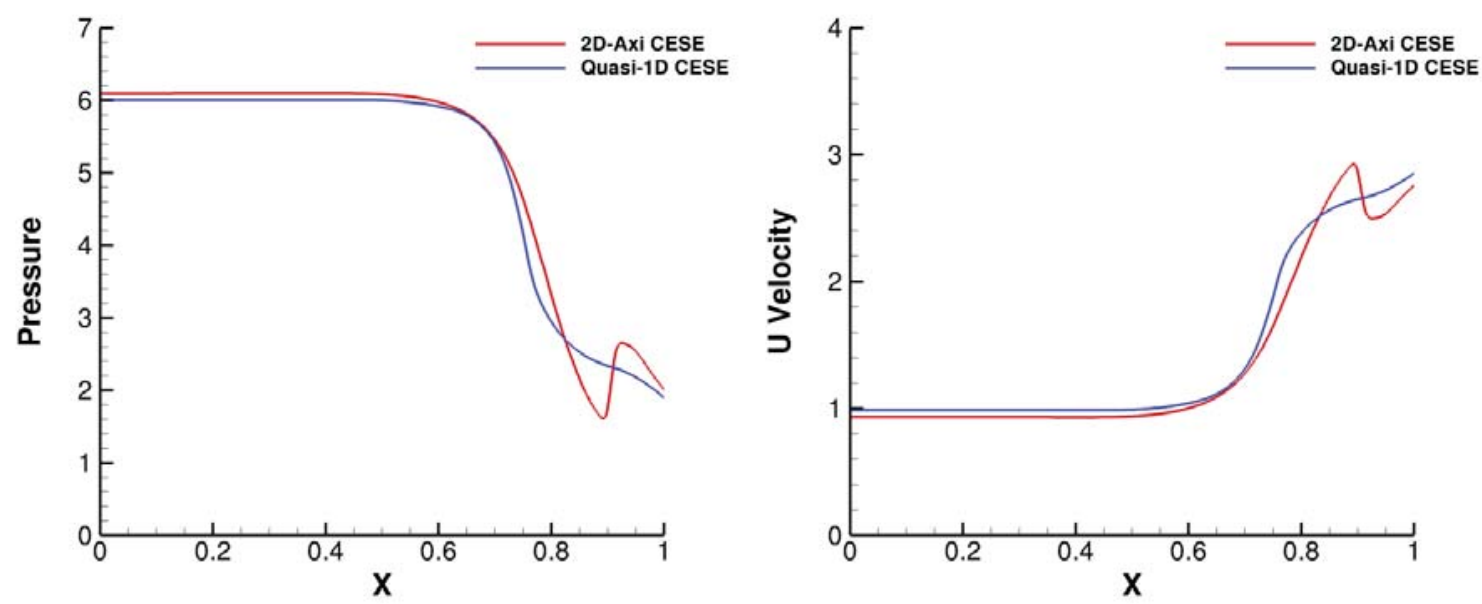

Figure 8. Pressure (left) and $u$ velocity (right) axial distributions for the primary duct.

6

American Institute of Aeronautics and Astronautics 


\section{B. Gross Thrust Computation}

While examining flow field quantities such as pressure and $u$ velocity can provide insight into the differences between the quasi-1D and 2D-axisymmetric solutions, further insight can gained by examining the computed gross thrust for the nozzle. Gross thrust is of particular interest as it is one of the main parameters used when designing/sizing a nozzle for integration with an aircraft propulsion system. Analytically, the gross thrust is defined over a control volume as:

$$
F=\int \rho u^{2} d A+\int\left(p-p_{\infty}\right) d A
$$

However, the gross thrust can be numerically computed by defining a control surface with a discrete stencil and numerically integrating the thrust along the stencil. For 2D-axisymmetric geometries, Eq. 8 becomes:

$$
F \approx \sum_{i=1}^{n-1} A_{i}\left(\frac{\rho_{i}+\rho_{i+1}}{2}\right)\left(\frac{u_{i}+u_{i+1}}{2}\right)^{2}+\left(\left(\frac{p_{i}+p_{i+1}}{2}\right)-p_{\infty}\right) A_{i}
$$

in which

$$
A_{i}=\pi\left(r_{i}^{2}-r_{i+1}^{2}\right)
$$

One potential flaw in Eq. 9 is that it will only compute quantities over the discretized area, which depending on how the stencil endpoints are chosen, might not represent the true area. This can be caused by having the stencil start and end nodes not on the true geometrical boundary. To mitigate the effect of the potential gaps at the nozzle walls, the computed gross thrust is corrected by the known geometrical area.

$$
F=F \frac{A_{\text {actual }}}{A_{\text {stencil }}}
$$

For computing the gross thrust, three different control surfaces were used and are shown in Fig. 9 through Fig. 11. Because Eq. 9 and Eq. 10 are specifically for 2D-axisymmetric geometries, Eq. 8 was computed directly at the nozzle exit for the quasi-1D solutions, with a representative control surface shown in Fig. 12.

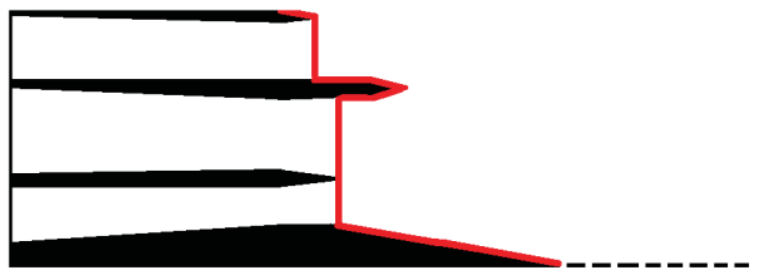

Figure 9. 2D-axisymmetric gross thrust control surface (shown in red), Configuration 1.

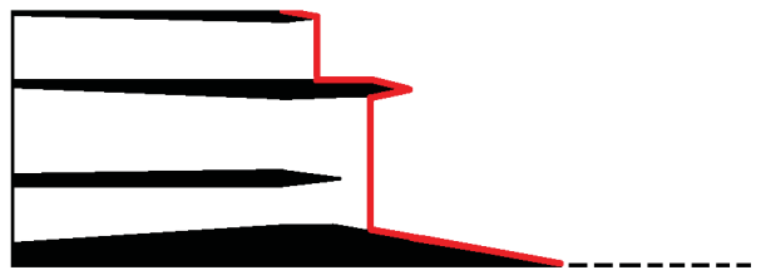

Figure 10. 2D-axisymmetric gross thrust control surface (shown in red), Configuration 1.5. 


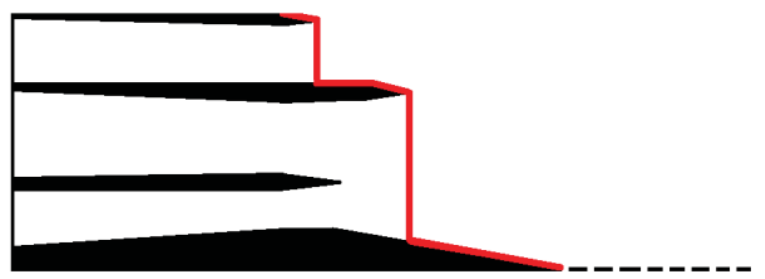

Figure 11. 2D-axisymmetric gross thrust control surface (shown in red), Configuration 2.

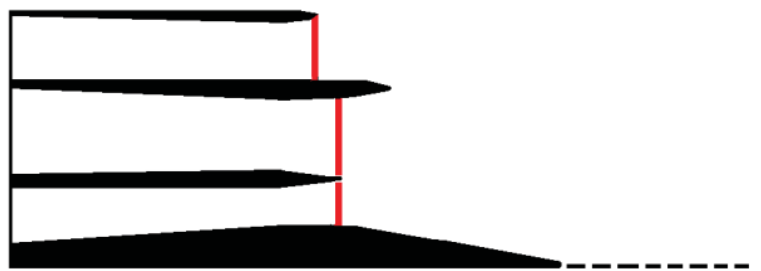

Figure 12. Quasi-1D gross thrust control surface (shown in red).

After developing the means to compute the gross thrust with different control surface definitions, a sensitivity study to the stencil resolution was performed. As a reminder, the stencil is the discrete points that represent the control surface, with a generic stencil concept shown in Fig. 13.

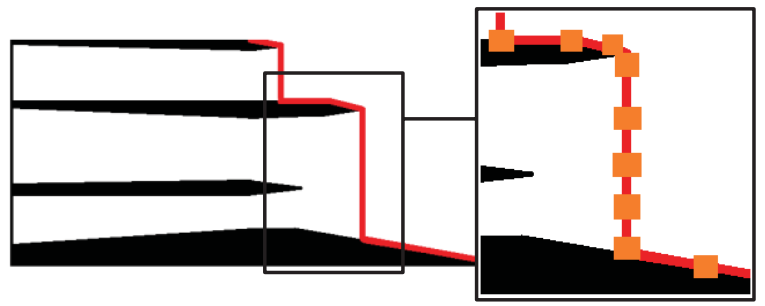

Figure 13. Sample control surface stencil. Note that the orange squares represent the discrete points along the control surface (shown in red).

Three separate 2D-axisymmetric steady-state solutions were used for the stencil resolution study: the Euler CESE and Wind-US solutions presented earlier and a 2D-axisymmetric viscous Wind-US solution which used the Menter's Shear Stress Transport (SST) ${ }^{15}$ turbulence model. The viscous Wind-US solution, denoted as Wind-US SST, was simulated to gain an insight to see if adding viscosity would reduce some of the convergence and conservation errors. The Wind-US SST simulation used a modified grid comprised of 401,000 structured cells with a max $y+$ of 0.49 . As a check, a grid resolution study was performed on the viscous grid in the same fashion as the Euler grid, the results of which are shown in Table 3. Just like the Euler grid, the viscous grid is shown to be approaching grid convergence and shows sufficient grid convergence at the fine level for the studies presented.

Table 3. Mass flow rates at various grid levels for the viscous Wind-US grid.

\begin{tabular}{|l|c|c|c|c|c|}
\hline \multicolumn{1}{|c|}{ Zone } & Coarse & Medium & Fine & \% Difference (C-M) & \% Difference (M-F) \\
\hline Zone 5 + Zone 4 (i=last) & 2.412 & 2.391 & 2.384 & 0.878 & 0.294 \\
\hline Zone 6 (i=1) & 2.412 & 2.391 & 2.384 & 0.878 & 0.294 \\
\hline Zone 6 (i=last) & 2.416 & 2.392 & 2.385 & 1.003 & 0.294 \\
\hline \% Mass Flow Gained & 0.166 & 0.042 & 0.055 & - & - \\
\hline
\end{tabular}

Figure 14 shows the sensitivity to the stencil resolution for the steady-state computed gross thrust (entire nozzle) and the combined mass flow rate for the inner and primary ducts. It can be seen that while all the mass flow rates converge to within $0.28 \%$ of each other, the gross thrusts using the viscous solution converge to a lower value than the Euler equivalents. This is due to low momentum flow developing in the boundary-layers of the viscous solution, which exploits the error derived from the gap between the starting/ending nodes of the stencil and the true geometric boundaries. Although Eq. 10 attempts to correct for this error, it assumes that the average values over the computed area are roughly equivalent to the average values over the gap areas. While this is true for Euler simulations, it is not necessarily true for viscous simulations. Examining Fig. 14 again, it is shown that all configurations are 
relatively converged with 60-80 nodes per stencil for the inner and primary ducts. This stencil resolution roughly equates to half the radial grid resolution. Although it is not clear whether this level of stencil resolution can be used for other nozzles flows, it will be used for the remainder of this paper.
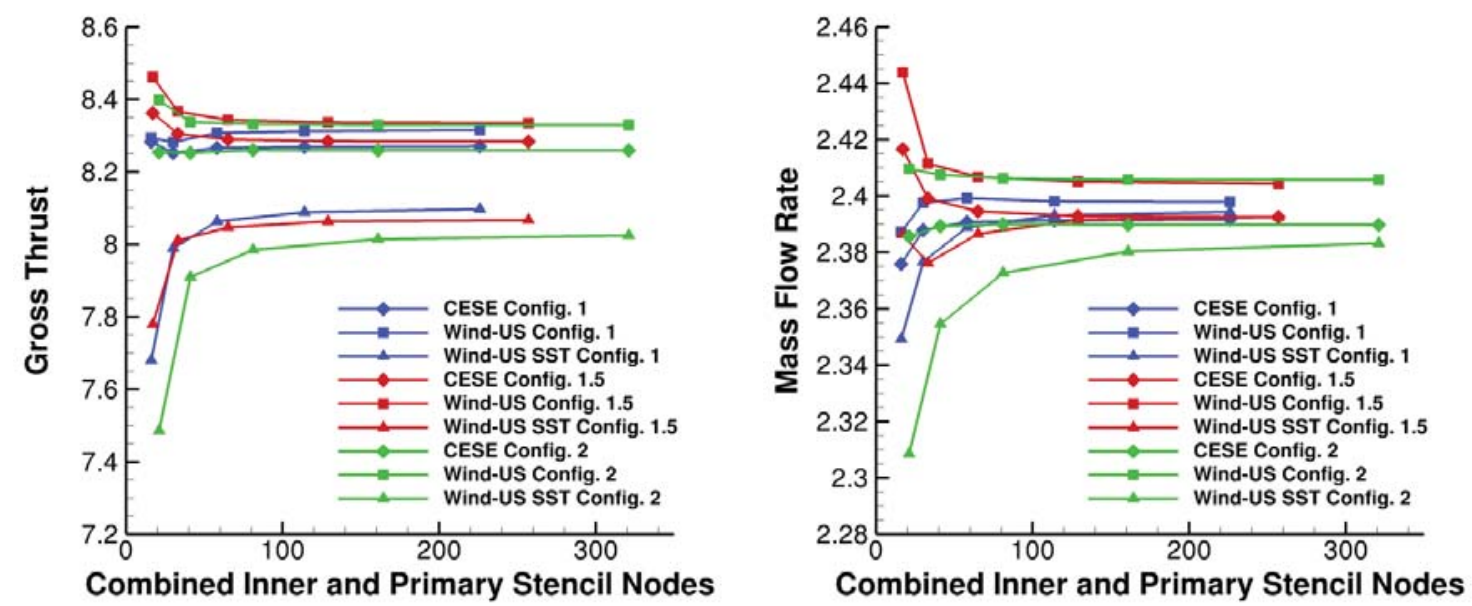

Figure 14. Gross thrust (left) and mass flow rate (right) sensitivities to stencil resolution.

\section{Steady-State Gross Thrust}

Table 4 shows the computed gross thrusts for various CESE Euler solutions and stencil configurations. Note that in the table, the percent difference was defined as:

$$
\% \text { Difference }=100 \times \frac{F-F_{\text {quas } i-1 D}}{F_{\text {quas } i-1 D}}
$$

It can be seen that the gross thrust computed using the $2 \mathrm{D}$-axisymmetric solution is within $1.46 \%$ of the gross thrust computed using the quasi-1D solution. However, the gross thrusts computed using the 2D-axisymmetric solution are consistently lower due to 2D effects such as oblique shock waves while all are within $0.36 \%$ of each other. This suggests that the computed gross thrust is not overly sensitive to the presented control surface definitions. However, this might not be the case when computing the unsteady gross thrust and will be explored further in the next section.

Table 4. CESE-Based Computed Gross Thrust (Euler solutions).

\begin{tabular}{|l|c|c|}
\hline \multicolumn{1}{|c|}{ Case } & Gross Thrust & \% Difference \\
\hline Quasi-1D & 8.383 & 0.00 \\
\hline 2D-Axi (Config. 1) & 8.266 & -1.39 \\
\hline 2D-Axi (Config. 1.5) & 8.290 & -1.10 \\
\hline 2D-Axi (Config. 2) & 8.261 & -1.46 \\
\hline
\end{tabular}

\section{Unsteady Gross Thrust}

For the unsteady simulations, a sinusoidal disturbance was introduced at the inlet of each duct. Since the CESE quasi-1D solution matched the analytical solution for the aeroacoustic benchmark, a similar incoming disturbance and boundary condition was used.

$$
\left[\begin{array}{l}
\rho^{\prime} \\
u^{\prime} \\
p^{\prime}
\end{array}\right]=\left[\begin{array}{l}
1 \\
1 \\
1
\end{array}\right] \varepsilon \sin (\omega t)
$$


where once again

$$
\left[\begin{array}{l}
\rho \\
u \\
p
\end{array}\right]=\left[\begin{array}{l}
\rho_{\text {steady }} \\
u_{\text {steady }} \\
p_{\text {steady }}
\end{array}\right]+\left[\begin{array}{c}
\rho^{\prime} \\
u^{\prime} \\
p^{\prime}
\end{array}\right]
$$

Each simulation was run at a different discrete frequency with a constant amplitude of 0.001 . The discrete frequencies are summarized in Table 5. Although this boundary condition had only been validated for quasi-1D simulations, it was applied both to the quasi-1D and 2D-axisymmetric simulations for consistency. Data from these simulations were then used to construct bode plots based on the input sinusoid and the unsteady component of the gross thrust.

$$
\begin{gathered}
\text { input }=0.001 \sin (\omega t) \\
\text { output }=F^{\prime}=F-F_{\text {steady }} \\
\text { Magnitude }=20 \log \left(\frac{\text { output }}{\text { input }}\right) \\
\text { Phase }=360 f \times\left(t_{\text {input }}-t_{\text {output }}\right)
\end{gathered}
$$

Although bode plots are presented, the reader is cautioned to view them in a qualitative light rather than a quantitative one as there is still discussion on how to properly model a disturbance boundary condition that results in meaningful dynamic data. Considering this caveat, bode plots comparing the quasi-1D and 2D-axisymmetric simulations for the primary duct are shown in Fig. 15 (with and without the natural time delay included in the phase shift). The natural time delay was defined as the time it would take for a signal to travel from the primary duct inlet to the primary duct exit control surface.

$$
\tau=\frac{x_{\text {outlet }}-x_{\text {inlet }}}{\left(u+\sqrt{\gamma \frac{p}{\rho}}\right)_{\text {avg }}}
$$

From Fig. 15 it can be seen that the 2D-axisymmetric unsteady computed gross thrust agrees well with the quasi1D unsteady computed gross thrust at the lower frequencies (with the exception of the lowest frequency) but diverges at the higher frequencies. This is particularly true when using control surface configurations 1.5 and 2 for the 2D-axisymmetric computation. Recall that the CFD solution is the same for all the 2D-axisymmetric gross thrusts with only the post-processing stencil changing. As a check, unsteady stencil resolution studies for configuration 1 and 2 are shown in Fig. 16. Note that the finest stencil resolutions are those that are shown in Fig. 15. It can be seen that unlike configuration 2 , the configuration 1 control surface is still highly sensitive to the stencil resolution. Although this seems contradictory to what Fig. 14 shows for steady-state, it is evident that a significant gap exists between the stencil for configuration 1 and the true geometry which the correction described in Eq. 11 does not appear to account for in the unsteady computation. 
Table 5. Pulsing Frequencies.

\begin{tabular}{|c|}
\hline Non-Dimensional Frequency \\
\hline 0.004 \\
\hline 0.04 \\
\hline 0.1 \\
\hline 0.2 \\
\hline 0.3 \\
\hline 0.4 \\
\hline 0.6 \\
\hline 0.8 \\
\hline 1.0 \\
\hline 1.5 \\
\hline 2.0 \\
\hline 2.5 \\
\hline 3.0 \\
\hline 4.0 \\
\hline
\end{tabular}
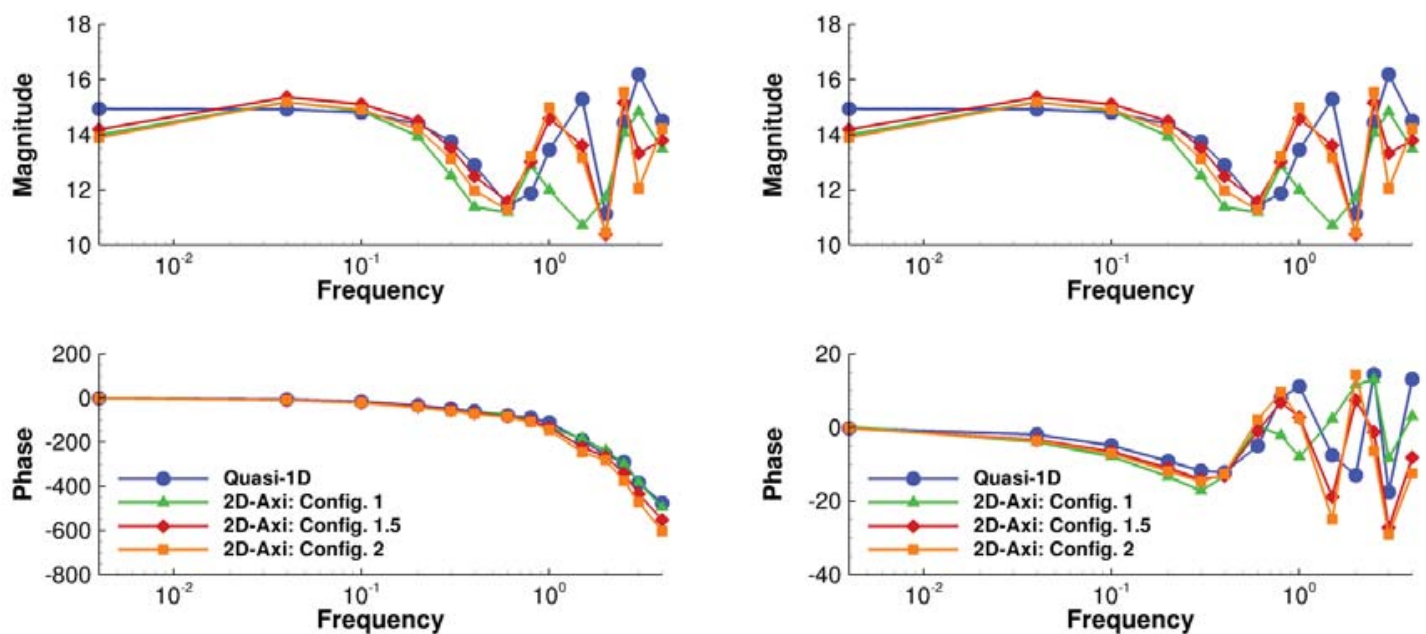

Figure 15. 2D-axisymmetric and quasi-1D primary duct bode plots with natural time delay (left) and without natural time delay (right).
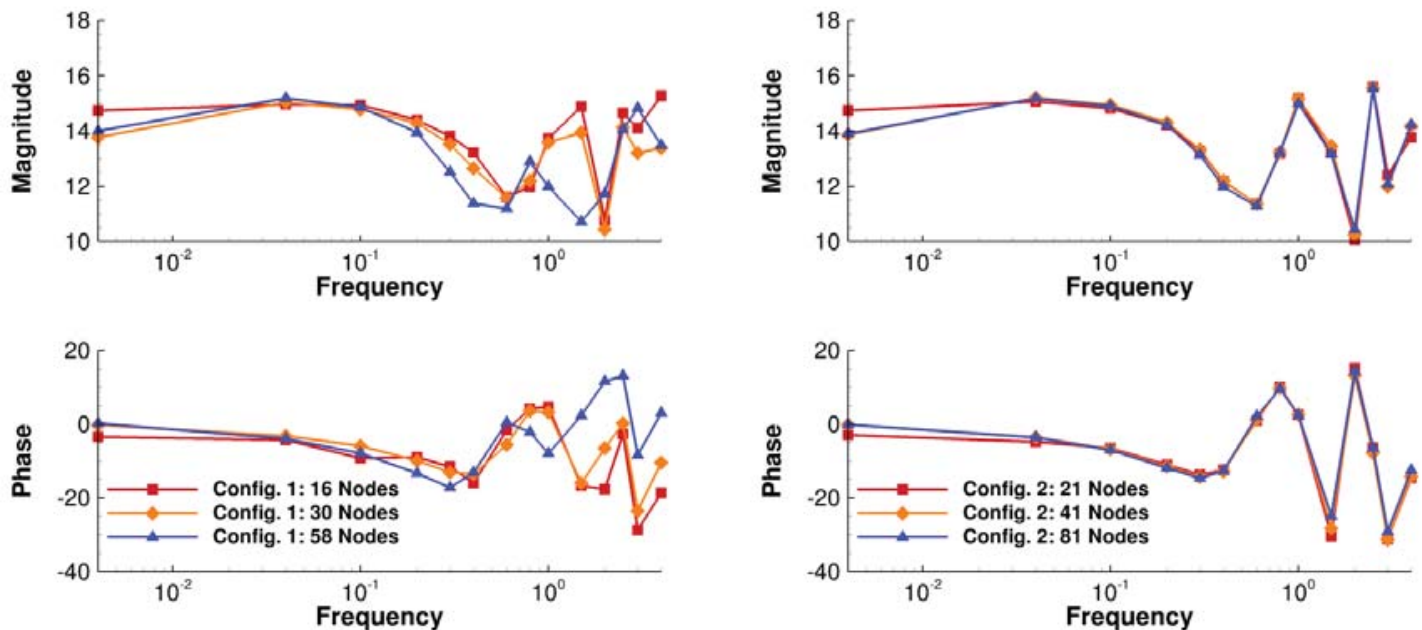

Figure 16. 2D-axisymmetric primary duct bode plot stencil resolution for Configuration 1 (left) and Configuration 2 (right). Note that the number of nodes is for both the inner and primary ducts combined.

11

American Institute of Aeronautics and Astronautics 


\section{Conclusions/Future Work}

Quasi-1D and 2D-axisymmetric numerical simulations were performed on a three-stream plug nozzle using the CESE method to aid in the development of a CFD-based nozzle flow model. First, steady-state flow field solutions were validated and showed excellent agreement with solutions derived from other codes and methods, demonstrating the capability of the CESE method. Second, a study was performed to explore the sensitivity of the control surface definition when computing gross thrust. Based on the study, it was shown that there was little sensitivity to the control surface definition itself. However, the study showed that the computed gross thrust was highly sensitive to the stencil resolution as well as the choice of the stencil end points. These were especially true for unsteady thrust computations partially due to the ineffectiveness of the stencil area correction on the unsteady computation. Third, the quasi-1D and 2D-axisymmetric solutions were compared. The steady-state solutions were shown to be similar, however, the quasi-1D solution failed to pick up the oblique shock wave at the downstream portion of the nozzle due to its limitations as a quasi-1D simulation. The solutions also varied when looking at the unsteady computed gross thrust, suggesting that the quasi-1D solution might not be sufficient for some system-level models. Because the disturbance boundary condition presented is most likely not suitable for real nozzle flows, potential future work includes the exploration of how to properly model a disturbance boundary condition such that meaningful dynamic data can be obtained from a CFD simulation. Also, additional unsteady simulations using the Wind-US code will be run to compare to the CESE-based unsteady solutions with a potentially updated disturbance boundary condition.

\section{Acknowledgments}

The authors would like to thank the NASA Fundamental Aeronautics Program for support with this research effort as well as Joseph Connolly for providing the quasi-1D analytical and MacCormack method solutions and Nicholas Georgiadis for thrust computation guidance.

\section{References}

${ }^{1}$ Chang, S. C., "The Method of Space-Time Conservation Element and Solution Element - A New Approach for Solving the Navier-Stokes and Euler Equations," Journal of Computational Physics, Vol. 119, Issue 2, July 1995, pp. $295-325$.

${ }^{2}$ Chang, S. C., Wang, X. Y., and Chow, C. Y., "The Space-Time Conservation Element and Solution Element Method - A New High-Resolution and Genuinely Multidimensional Paradigm for Solving Conservation Laws," Journal of Computational Physics, Vol. 156, Issue 1, November 1999, pp. 89-136.

${ }^{3}$ Wang, X. Y., Chang, S. C., "A 2D Non-splitting Unstructured-triangular-mesh Euler Solver Based on the Method of SpaceTime Conservation Element and Solution Element," Computational Fluid Dynamics Journal, Vol. 8, No. 2, 1999 , pp. 326-340.

${ }^{4}$ Loh, C. Y., Hultgren, L. S., and Chang, S. C., "Wave Computation in Compressible Flow Using Space-Time Conservation Element and Solution Element Method," AIAA Journal, Vol. 39, No. 5, May 2001, pp. 794-801.

${ }^{5}$ Loh, C. Y., and Zaman, K. B. M. Q., "Numerical Investigation of Transonic Resonance with a Convergent-Divergent Nozzle," AIAA Journal, Vol. 40, No. 22, December 2002, pp. 2393-2401.

${ }^{6}$ Chang, C. L., "Time-Accurate, Unstructured-Mesh Navier-Stokes Computations with the Space-Time CESE Method," AIAA Paper 2006-4780, July 2006.

${ }^{7}$ MacCormack, R. W., "The Effect of Viscosity in Hypervelocity Impact Cratering,” AIAA Paper 69-354, April 1969.

${ }^{8}$ Anderson, J. D., Computational Fluid Dynamics: The Basics with Applications, McGraw-Hill, New York, NY, 1995.

${ }^{9}$ Towne, C., "Wind-US User's Guide, Version 2.0," NASA/TM 2009-215804, October 2009.

${ }^{10}$ Georgiadis, N., and Papamoschou, D., “Computational Investigations of High-Speed Dual-Stream Jets,” AIAA Paper 20033311, May 2003.

${ }^{11}$ Roe, P. L., "The Use of the Riemann Problem in Finite-Difference Schemes," Lecture Notes Physics, Vol. 141, SpringerVerlag, New York, pp. 354-359.

${ }^{12}$ Roe, P. L., "Approximate Riemann Solvers, Parameter Vectors and Difference Schemes," Journal of Computational Physics, Vol. 43, pp. 357-372.

${ }^{13}$ Dahl, M., "Third Computational Aeroacoustics (CAA) Workshop on Benchmark Problems," NASA/CP 2000-209790, August 2000.

${ }^{14}$ Wang, X. Y., Chang, S. C., and Jorgenson, P., "Prediction of Sound Waves Propagating Through a Nozzle Without/With a Shock Wave Using the Space-Time CE/SE Method," NASA/TM 2000-209937, March 2000.

${ }^{15}$ Menter, F. R., "Two-Equation Eddy Viscosity Turbulence Models for Engineering Applications," AIAA Journal, Vol. 32, No. 8, 1994, pp. 1598-1605. 